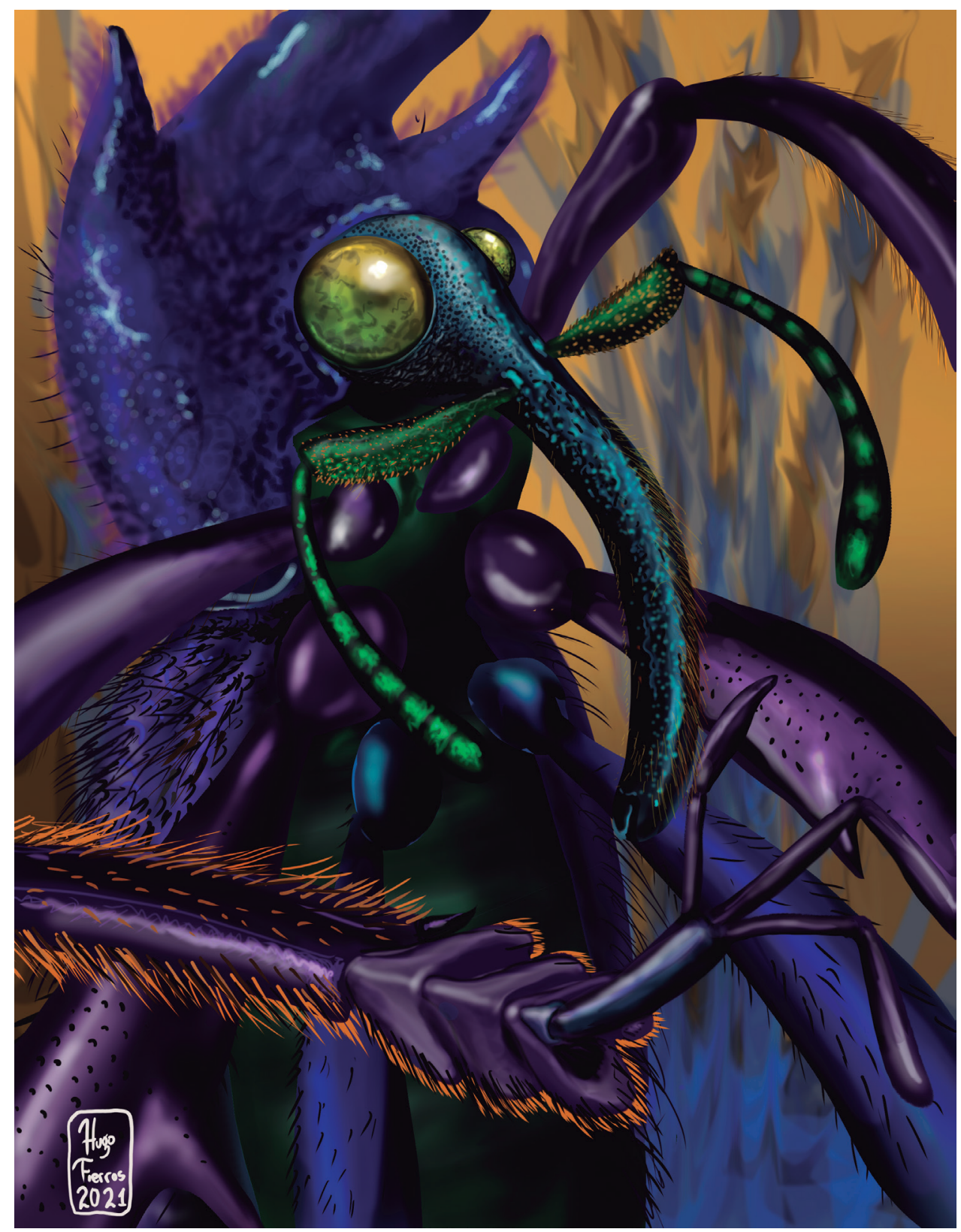

Dugesiana, Año 28, No. 2, (julio-diciembre, segundo semestre de 2021), es una publicación semestral, editada por la Universidad de Guadalajara, a través del Centro de Estudios en Zoología, por el Centro Universitario de Ciencias Biológicas y Agropecuarias. Camino Ramón Padilla Sánchez \# 2100, Nextipac, Zapopan, Jalisco, Tel. 37771150 ext. 33218, http://148.202.248.171/dugesiana/index.php/DUG/index, glenusmx@gmail.com. Editor responsable: José Luis Navarrete-Heredia. Reserva de Derechos al Uso Exclusivo 04-2009-062310115100-203, ISSN: 2007-9133, otorgados por el Instituto Nacional del Derecho de Autor. Responsable de la última actualización de este número: José Luis Navarrete-Heredia, Editor y Ana Laura González-Hernández, Asistente Editorial. Fecha de la última modificación 1 de julio de 2021, con un tiraje de un ejemplar.

Las opiniones expresadas por los autores no necesariamente reflejan la postura del editor de la publicación.

Queda estrictamente prohibida la reproducción total o parcial de los contenidos e imágenes de la publicación sin previa autorización de la Universidad de Guadalajara. 


\title{
Desde la Patagonia al Valle de Anáhuac
}

\section{From la Patagonia to Valle de Anáhuac}

\author{
Juan J. Morrone \\ Museo de Zoología 'Alfonso L. Herrera', Departamento de Biología Evolutiva, Facultad de Ciencias, Universi- \\ dad Nacional Autónoma de México (UNAM), 045010 Ciudad de México, México. morrone@ciencias.unam.mx, \\ ORCID: https://orcid.org/0000-0001-5566-1189
}

Hace casi 50 años me enamoré de la biología evolutiva, gracias a mi maestra de sexto grado de la primaria que me impulsó a leer El origen de las especies. Nunca imaginé que años más tarde terminaría siendo profesor de biología en la Universidad Nacional Autónoma de México. Con motivo del homenaje que me hace la revista Dugesiana, a través de su director José Luis Navarrete, me gustaría contar de manera breve este recorrido, incluyendo algunas reflexiones personales. Espero que los lectores jóvenes sepan disculpar el tono anecdótico y que mis coetáneos disfruten leer acerca de mis experiencias.

Hace 58 años nací en Quilmes, Argentina. Mis padres, Juan Carlos Morrone y Lidia Isabel Lupi (Fig. 1), me dieron la mejor educación posible. En la década de 1960 y comienzos de la década de 1970 Argentina aún contaba con una educación pública de excelencia, herencia de algunos gobiernos liberales del siglo 19 y comienzos del 20. (Aún no habían llegado los gobiernos neoliberales y los populismos pseudoprogresistas que habrían de destruir la educación pública.) Recuerdo con especial afecto la Escuela 19 "Domingo Faustino Sarmiento", donde cursé la primaria, y la Escuela Normal Mixta de Quilmes "Almirante Guillermo Brown", donde hice la secundaria. En la primaria tuve una maestra excepcional, Luisa Kruchosky de Escobar, que impulsó mi interés temprano por las ciencias naturales. (Mi amiga Irina Podgorny y yo hemos prometido alguna vez dedicarle un libro.) Ciencias fisico-químicas y naturales de Alberto Fesquet fue una lectura fascinante, que estimuló mi pasión por los seres vivos. En los primeros años de la escuela secundaria, mi profesor Norberto Traverso impartió los cursos de Botánica y Zoología, que me resultaron muy interesantes. En el ciclo superior de la secundaria (la "prepa") cursé el Bachillerato Biológico, donde la profesora Lidia Hurtado de Quijano me impulsó a seguir estudios universitarios de biología en el Museo de La Plata. (Algunas veces dudé en estudiar veterinaria, letras o filosofía, pero la biología fue mi elección final.)

Entre 1981 y 1985 estudié la carrera de Biología con orientación zoológica en la Facultad de Ciencias Naturales y Museo de la Universidad Nacional de La Plata (Fig. 2). Allí desarrollé amistades entrañables, hoy en día desperdi- gados por el mundo, pero que siguen presentes a través de Facebook. Tuve maestros que me marcaron para siempre, algunos por su notable capacidad docente y otros, lamentablemente, por carecer de ella. Debo a los primeros, entre quienes se encuentran Jorge Crisci, Miguel Manceñido, Zulma Ageitos de Castellanos, Raúl Arámburu, Ricardo Ronderos, Julia Vidal Sarmiento, Estela Lopretto, Norma Díaz y Marta Loiácono, mi interés por la sistemática, la biogeografía y la evolución. Debo a los segundos, cuyos nombres por pudor omito, una parte de mi incapacidad para apreciar la ecología.

Cuando terminé la carrera inicié mis estudios de doctorado bajo la dirección de Jorge Crisci y Analía Lanteri, con la revisión sistemática de un grupo de gorgojos (Coleoptera: Curculionidae) de la Patagonia. Debo mucho a Jorge y Analía, sobre todo la libertad con que me dejaron trabajar y el modo en que me impulsaron a ser un investigador independiente. Además, tuve una importante interacción con Sergio Roig-Juñent, Susana Freire y María Marta Cigliano, quienes en ese entonces también eran estudiantes de doctorado, y con quienes compartí estimulantes discusiones. (Recuerdo que con María Marta nunca dejábamos de molestar a Sergio por su pasión por La gènese des faunes terrestres de René Jeannel.) Nelly Vittet, la secretaria del Laboratorio de Sistemática y Biología Evolutiva, me enseñó a escribir con pulcritud y a revisar mis escritos con esmero rayano en la obsesión. (Cuando fui editor de la Revista de la Sociedad Entomológica Argentina pasé tardes enteras con Nelly corrigiendo manuscritos y pruebas de página, desarrollando un odioso "ojo clínico" para detectar errores.) Para mi trabajo de tesis doctoral trabajé con cientos de ejemplares de una veintena de colecciones, así como de otros (pocos) recolectados por mí. En los viajes de recolecta a la Patagonia, en el sur de Argentina y Chile, me empezó a interesar la biogeografía. De estos viajes, recuerdo largas pláticas con Sergio Roig-Juñent, Emilio Maury y Liliana Katinas, en que me comenzó a interesar la biota patagónica y sus relaciones con las biotas de otros continentes australes. Luego establecí correspondencia con Robin Craw, de Nueva Zelanda, y nació mi interés en la panbiogeografía, el cual se incrementaría a partir de la visita de Jorge Crisci a Nueva 
Zelanda y su interacción con Robin Craw y Michael Heads. (Una consecuencia desafortunada de esa visita fue que nos introdujeron a la deconstrucción de Jacques Derrida, la cual tardé años en descubrir que era completamente estéril.)

A principios de la década de 1990 realicé mi posdoc en el American Museum of Natural History de Nueva York, donde interactué con Norman Platnick, Jim Carpenter, Toby Shuh y Lee Herman, así como con Pablo Goloboff, quien en ese momento se encontraba allí haciendo su doctorado, y su esposa Claudia Szumik. En el Museo consolidé mi perspectiva filogenética, bajo la variante del "cladismo de patrón", que adopté con fervor, pero abandoné años más tarde cuando éste derivó a posiciones antievolutivas, que creo inaceptables. (¡A Aradezco mucho a Steve Farris el abrirme los ojos y permitirme superar este error juvenil!)

A lo largo de 15 años fui docente de Introducción a la taxonomía y de Biogeografía en la Facultad de Ciencias Naturales, comenzando en 1983 como ayudante alumno, más tarde como ayudante graduado y jefe de trabajos prácticos, y finalmente como profesor adjunto. En 1998 gané la plaza de profesor asociado de Zoología general y todo parecía encaminado a mi estabilidad académica en el Museo de La Plata. Sin embargo, la situación habría de cambiar drásticamente. Unos años antes, en 1994, Jorge Llorente-Bousquets me había invitado a participar como profesor del Diplomado de Actualización en Biología Comparada Contemporánea, que se impartió en la Facultad de Ciencias de la UNAM, auspiciado por la Comisión Nacional para el Conocimiento y Uso de la Biodiversidad (CONABIO). En los años siguientes, volvió a invitarme a los siguientes diplomados, que se impartieron en el Centro de Investigaciones Científicas de Quintana Roo en Chetumal (1995), el Instituto Politécnico Nacional en Ciudad de México (1996) y la Universidad Autónoma de Nuevo León en Monterrey (1997). Finalmente, en 1998, gracias a las gestiones de Jorge y del director de la Facultad de Ciencias, Rafael Pérez Pascual, así como de una beca patrimonial de CONACyT, me ofrecieron una plaza de profesor de carrera en el área de Sistemática y Biogeografía. Cinco años más tarde obtuve la ciudadanía mexicana, con lo cual se consolidó de manera definitiva la relación afectiva con mi patria. Cuando alguien me cuestiona el acento que aún delata mi origen austral, contesto con la frase de Chabela Vargas, quien había nacido en Costa Rica, y alguna vez expresara rotundamente: "los mexicanos nacemos donde nos da la chingada gana".

En el último cuarto de siglo he venido desarrollando una línea de investigación y docencia en biogeografía evolutiva y sistemática filogenética, en el Museo de Zoología 'Alfonso L. Herrera'. Allí cuento entre mis colegas y amigos a Jorge Llorente, Tania Escalante, Roxana Acosta, Isabel Vargas, Armando Luis, Adolfo Navarro, Luis Sánchez, Blanca Hernández, Livia León, Oscar Flores, Rosario Mata, Erick García, Alejandro Gordillo, Leticia Ochoa y Adrián Nieto. Todos ellos me han ayudado de una u otra forma a mi trabajo y les estoy sumamente agradecido. Fuera del Museo, además, cuento con colegas y amigos en los otros depar- tamentos de la Facultad de Ciencias, en las Facultades de Estudios Superiores Zaragoza e Iztacala, y en los institutos de Biología, Ecología, Geología y Ciencias del Mar y Limnología. La UNAM es un universo complejo y maravilloso, tanto en lo material como en lo humano. Pertenecer a este universo, dentro de ese otro universo complejo y maravilloso que es mi patria de elección, es una bendición.

Mi investigación en biogeografía se centra fundamentalmente en una pregunta: ¿cómo es que los seres vivos llegaron a distribuirse en las áreas donde se encuentran? Tanto en mis trabajos iniciales en la Patagonia argentino-chilena como en los más recientes en la Zona de Transición Mexicana, he intentado responder esta pregunta. Inicialmente, tuve una perspectiva estrictamente vicariancista, desdeñando las explicaciones dispersalistas como narraciones especulativas y sin sustento científico. Esta perspectiva vicariancista tenía dos implementaciones metodológicas: por un lado, la panbiogeografía desarrollada por Léon Croizat, Robin Craw, Michael Heads y John Grehan; y por otro, la biogeografía cladística, desarrollada por Gareth Nelson, Norman Platnick y Donn E. Rosen. Creo que los continentes australes (América del Sur, Nueva Zelanda y Australia), que alguna vez formaron parte del supercontinente Gondwana, son particularmente adecuados para ser analizados desde la perspectiva vicariancista. En México, a partir de mi interacción con Gonzalo Halffter, del Instituto de Ecología, A.C. de Xalapa, comencé a interesarme más en el proceso de dispersión, en particular por su indudable relevancia en el desarrollo biótico de la Zona de Transición Mexicana, la zona de superposición entre las regiones Neártica y Neotropical. Para mi comprensión de la complejidad de la Zona de Transición Mexicana algunas pláticas con Pedro Reyes-Castillo y Mario Zunino también fueron fundamentales. A lo largo de los años fui descubriendo que algunos biogeógrafos como Halffter, Osvaldo Reig y Jay Savage habían propuesto superar la disyuntiva vicariancista-dispersalista. En la última década terminé desarrollando una perspectiva integrativa, ya esbozada en un artículo con Jorge Crisci en 1995, que considera que la vicarianza y la dispersión son procesos igualmente relevantes para explicar la evolución biótica. Esto me hace una especie de "bicho raro", pues los panbiogeógrafos me consideran un dispersalista, mientras que para los dispersalistas soy un vicariancista sin remedio.

En sistemática he continuado trabajando con Curculionidae, a los que he incorporado el estudio de los Siphonaptera (pulgas) y de otros grupos de Coleoptera. En los últimos años, he comenzado a trabajar más intensamente con Curculionidae del suelo y la hojarasca, los que son generalmente ciegos, en particular con mi estudiante y ahora colega Manuel Barrios (Fig. 3). Con mis estudiantes he llevado a cabo revisiones sistemáticas de numerosos taxones, tratando de incrementar el conocimiento de la biodiversidad de nuestro país. Hace unos meses impartí una plática en el Congreso de la Asociación Mexicana para la Sistemática de Artrópodos, en la cual intenté reflejar mi filosofía del trabajo sistemático, a partir de los siguientes 12 pasos que 
permitirían a quien los siga ser un "sistemático feliz":

(1) Disfrutar la naturaleza.

(2) Disfrutar la recolecta de organismos.

(3) Disfrutar el descubrimiento de especies nuevas.

(4) Disfrutar la búsqueda bibliográfica.

(5) Disfrutar la revisión de colecciones.

(6) Disfrutar la elaboración de revisiones sistemáticas.

(7) Disfrutar el trabajo de laboratorio.

(8) Disfrutar la reconstrucción de relaciones filogenéticas.

(9) Disfrutar los análisis evolutivos.

(10) Disfrutar la publicación de artículos.

(11) Disfrutar la enseñanza de la sistemática.

(12) Disfrutar la divulgación de la sistemática.

Además de la biogeografía y la sistemática, existen otras áreas que me interesan, como la evolución, la conservación y la historia de la biología. En los últimos años y sobre todo debido al encierro por la pandemia de COVID-19, he trabajado más intensamente en esta última área, estando mi interés enfocado particularmente en la historia y filosofía de la biogeografía evolutiva. Si bien muchos científicos ven con recelo la investigación histórica, creo que para comprender a cabalidad una disciplina es preciso conocer su desarrollo histórico y las principales discusiones filosóficas relacionadas con sus ideas principales. Mi interés por la historia ha sido estimulado también por influencia de Ana Barahona, Edna Suárez, Rosaura Ruiz y Jorge Llorente.

Un área en la que querría haber trabajado más es en divulgación de la ciencia. He hecho algunas contribuciones acerca de la crisis de la sistemática filogenética, la biogeografía evolutiva, la biodiversidad, los jardines zoológicos, los animales domésticos, la entomología forense y los animales fantásticos. Creo que en la actualidad la divulgación de la ciencia posee una importancia fundamental, debido a que existen numerosas fuerzas que conspiran contra la ciencia, tanto en nuestro país como en el resto del mundo, como el terraplanismo, el creacionismo, el movimiento antivacunas, la homeopatía y el movimiento antitransgénicos. Si algo nos ha enseñado la pandemia del COVID-19 es que sin ciencia no tenemos futuro, y la divulgación de la ciencia es importante para que la gente aprecie el pensamiento científico y no sienta que la ciencia es inútil y hasta peligrosa.

Más que un investigador me considero un maestro. He impartido numerosos cursos de licenciatura y posgrado y he dirigido las tesis de varios estudiantes (Fig. 4). Los trabajos sistemáticos de mis estudiantes corresponden a diversas familias, como Curculionidae (Paula Posadas, María Ventura Rosas, Alberto Romo, Perla Cuevas, Manuel Barrios, Kevin Cortés y Julián Mendivil), Staphylinidae (Juan Márquez y Julieta Asiain), Melolonthidae (Jocelyn Jacinto), Passalidae (Enio Cano), Buprestidae (Angélica Corona), Chrysomelidae (Miguel Eligio), Brentidae (Beatriz Acevedo), Elmidae (Josefina Curiel), Hystrichopsyllidae (Roxana Acosta), Formicidae (César Vázquez), Geocoridae (Laura Rengifo), Alpheidae (Margarita Hermoso), Sicariidae (Isabel Navarro), Poecilidae (Leticia Huidobro) y Colubridae (Carla Bessa).
En cuanto a los análisis biogeográficos de mis alumnos, los taxones estudiados también han sido muy diversos: Curculionidae (Perla Cuevas y Alberto Romo), Siphonaptera (Ana Gutiérrez), Reduviidae (Laura Rengifo), Aeglidae (Georgina Tumini), Theraphosidae (Carlos Perafán), Gastropoda (Gabriel Aguilar), Aves (Esperanza Álvarez), Mammalia (Tania Escalante, Gabriela García-Marmolejo y Hugo Albornoz), plantas (Bárbara Larraín, Amparo Echeverry, Niza Gámez y Alfredo Padró) y taxones varios (Violeta Corral, Patricia García-Navarrete y Viridiana Lizano). Además, he participado en cientos de comités tutorales y de candidatura al grado de doctor. De mis estudiantes he aprendido mucho, pues interactuar con ellos me ha llevado a intentar ser más claro al explicar conceptos complejos, me ha obligado a mantenerme actualizado y me ha enseñado a cultivar la paciencia (en especial al leer las versiones preliminares de sus manuscritos en inglés). Además, mis exalumnos con el tiempo se han convertido en colegas y luego en mis maestros, destacándose en particular Tania Escalante, con quien he compartido numerosas publicaciones. Por otra parte, a lo largo de estos años he recorrido varios países de América Latina para impartir cursos de biogeografía (Fig. 5), y guardo un enorme afecto por los estudiantes que asistieron a ellos. Nunca podremos predecir las consecuencias de los cursos. El año pasado, en el Congreso Chileno de Entomología, en Concepción, me reencontré con una alumna de un curso que impartí hace años en Uruguay y me agradeció porque gracias a mi curso ella había conocido a su esposo.

Otro aspecto que me gustaría destacar de mi labor docente se debe a la influencia de Jorge Llorente, quien hace años me inculcó la pasión por escribir libros de texto en español. Ya sea solo o con Jorge, David Espinosa, Tania Escalante y Oscar Flores, he podido publicar varios libros de texto de sistemática y biogeografía. Al respecto la UNAM ha sido muy generosa al proporcionar el apoyo económico necesario. Para mí es una de las experiencias más gratificantes que alguien me escriba para decirme que utilizó uno de mis libros en una clase de sistemática o biogeografía.

Mi principal pasatiempo es la lectura. A lo largo de los años he acumulado varios miles de libros, entre los cuales me refugio para disfrutar su lectura y alejarme del mundanal ruido. Jorge Luis Borges, Walt Whitman, Ray Bradbury, Juan Rulfo, Octavio Paz, Gabriel García Márquez, Pablo Neruda, James Joyce y Marguerite Yourcenar se encuentran entre mis autores predilectos. Pedro Páramo es mi libro preferido y acumulo varios estudios críticos que me han ayudado a valorar más cabalmente su grandeza. Tengo también una afición especial por la filosofía, la política, la teología y la bioética. De esta última, gracias a interesantes pláticas con Ángeles Cancino, poseo una cantidad importante de libros.

Para finalizar este recorrido, me gustaría agradecer a mi familia, maestros, colegas, estudiantes y amigos por casi 60 años de experiencias compartidas. Sus nombres aparecen en el texto de esta contribución. Muchas disculpas si me olvidé de alguno. Agradezco a Adrián Fortino por un cuarto 
de siglo de compartir mi vida. Como alguna vez expresara la poetisa chilena Violeta Parra: ¡Gracias a la vida que me ha dado tanto!

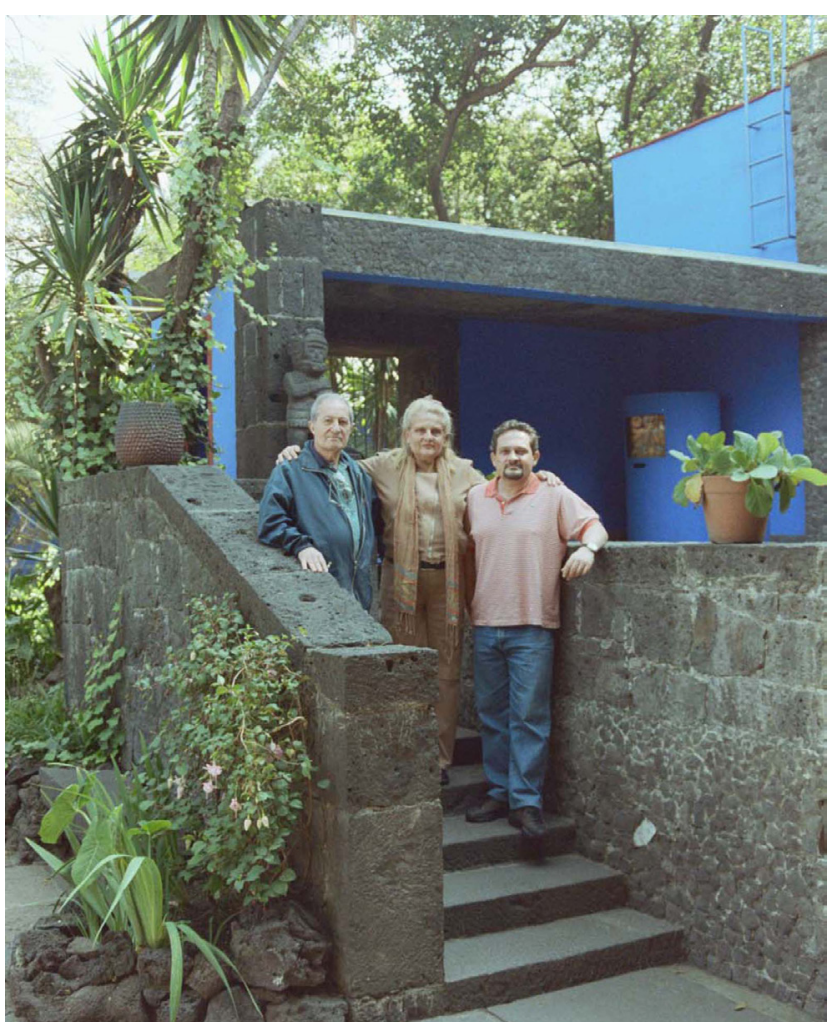

Figura 1. Junto con mis padres, visitando la casa de Frida Kahlo, Ciudad de México (año 2007).

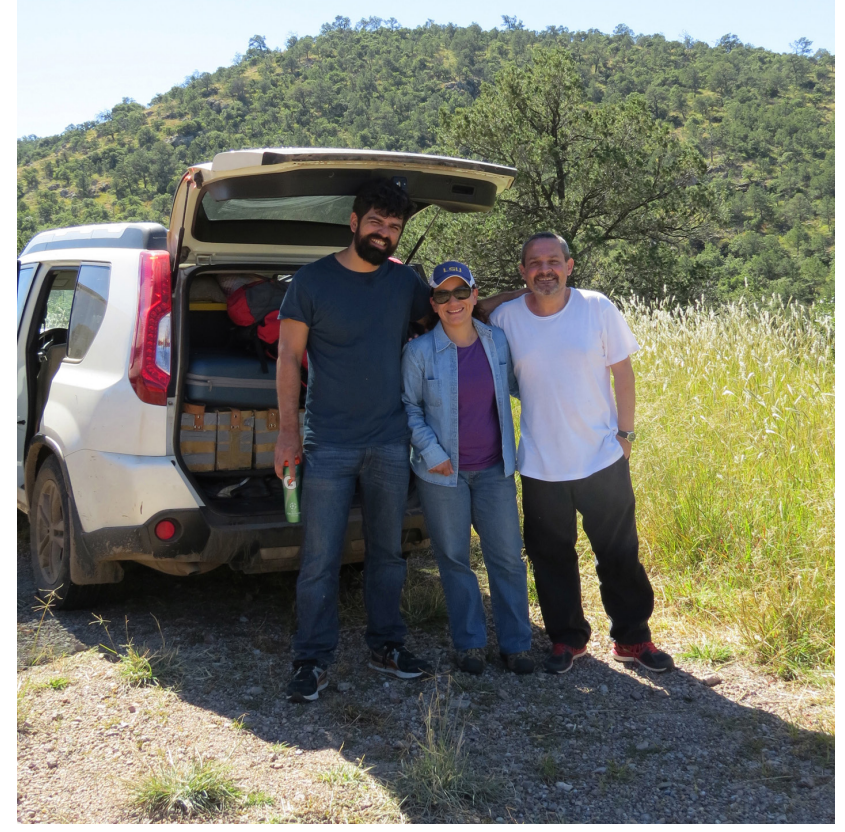

Figura 3. Viaje de recolecta a Chihuahua con Roxana Acosta, Manuel Barrios y Jesús Fernández (quien tomó la fotografía, año 2016).

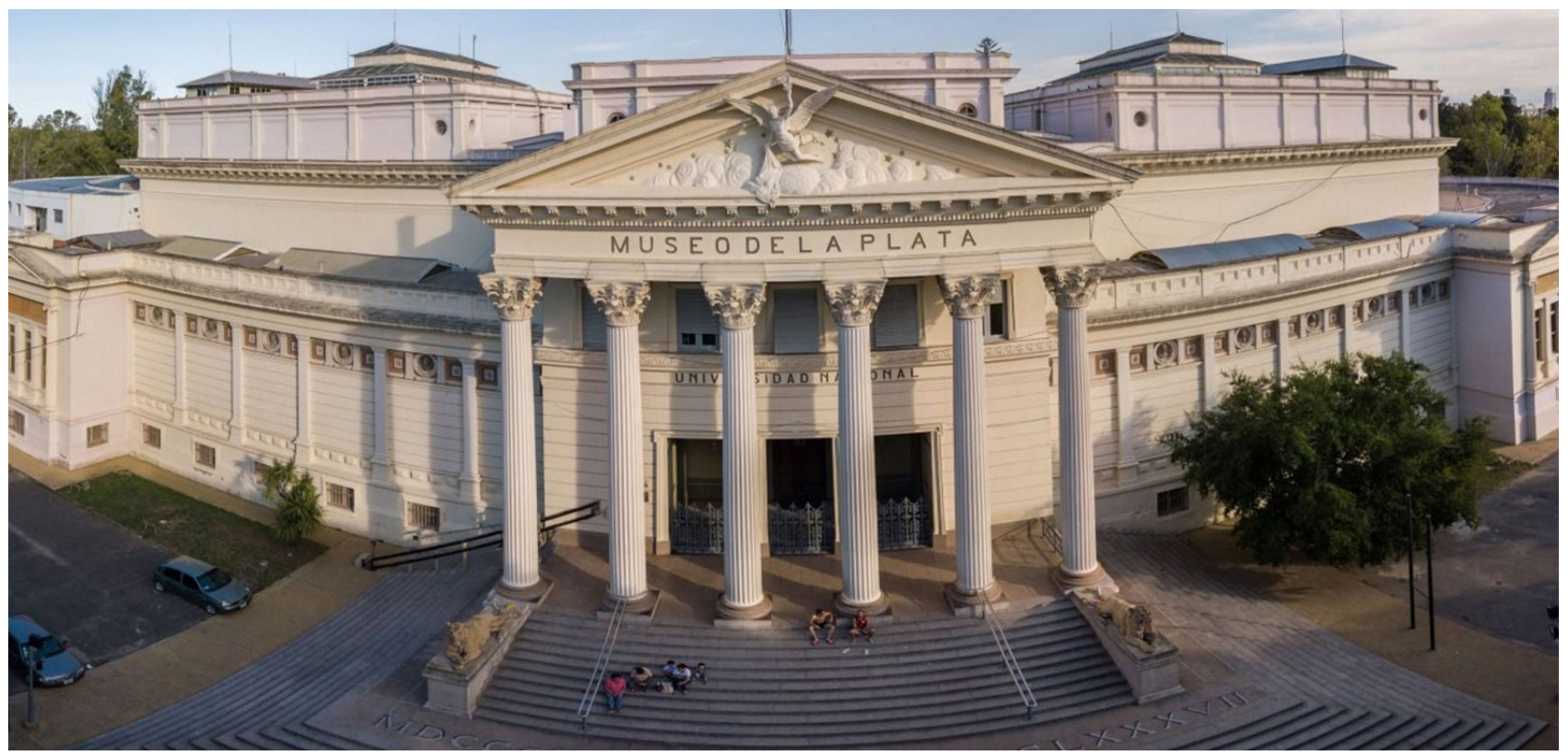

Figura 2. Museo de La Plata, La Plata, Argentina. 


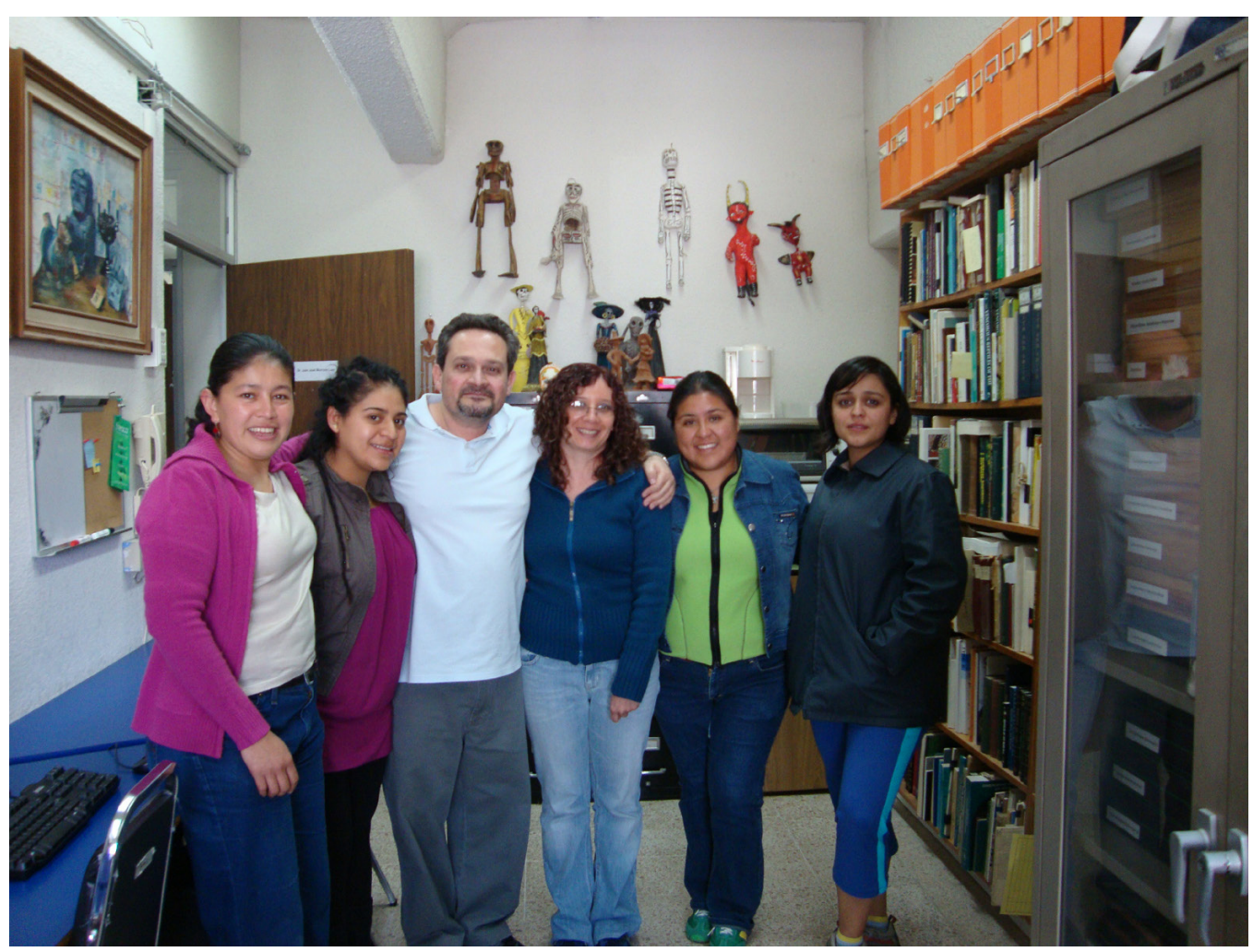

Figura 4. Junto con algunas estudiantes en mi cubículo del Museo de Zoología.

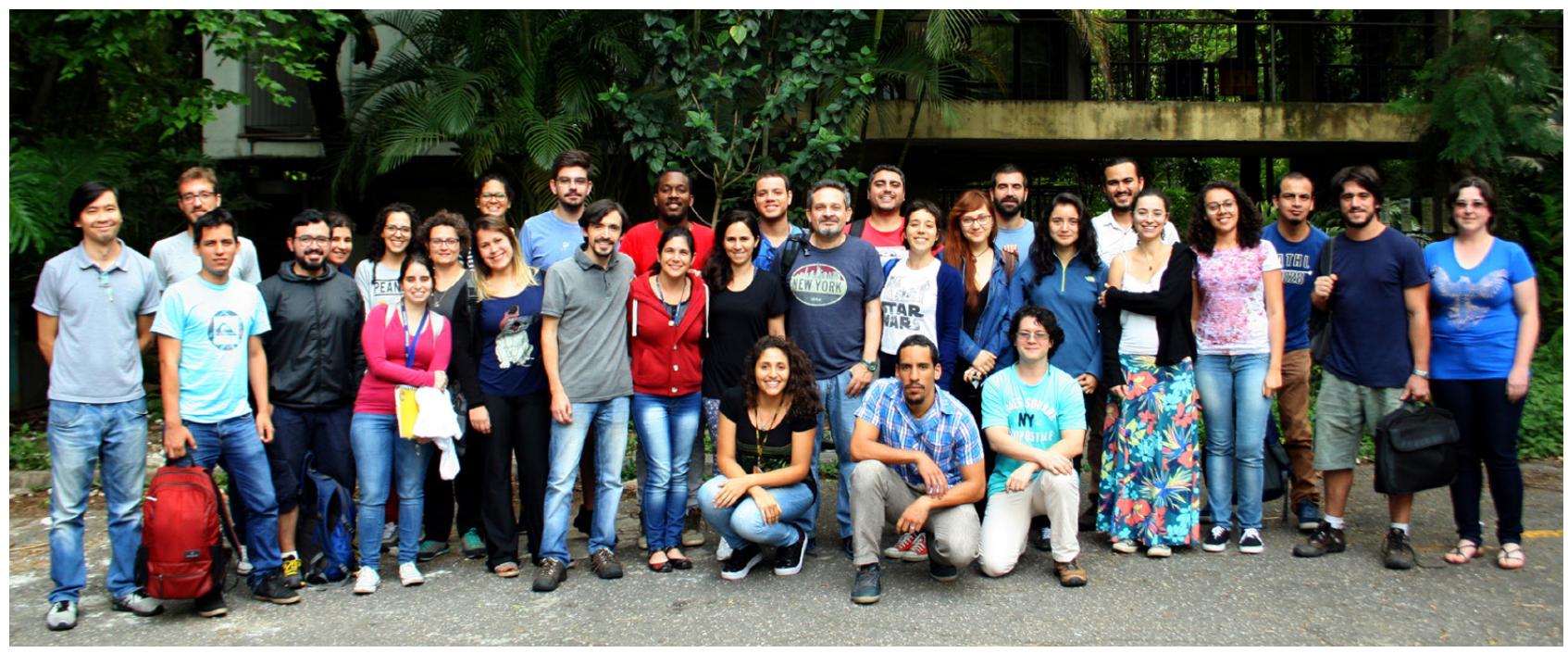

Figura 5. Con los estudiantes del curso impartido en el Instituto de Biociências, Universidad de São Paulo, Brasil (año 2017). 